\title{
ARTICLE \\ Cesarean section increases sensitivity to oxazolone-induced colitis in C57BL/6 mice
}

\author{
Line Fisker Zachariassen ${ }^{1}$, Axel Kornerup Hansen ${ }^{1}$, Lukasz Krych², Dennis Sandris Nielsen ${ }^{2}$, Thomas Lindebo Holm³ \\ Peter Tougaard ${ }^{4,5}$ and Camilla Hartmann Friis Hansen ${ }^{1}$
}

\begin{abstract}
Children born by cesarean section (CS) have an increased risk of developing inflammatory bowel disease (IBD), possibly due to skewed microbial colonization during birth and consequently impaired bacterial stimulation of the developing immune system. The aim of this study was to investigate the association between CS and experimental colitis in a murine model of IBD. It was hypothesized that CS aggravates colonic inflammation due to a change in gut microbiota (GM) composition. C57BL/6 mice, delivered by CS or vaginal delivery (VD), were intra-rectally challenged with oxazolone at 8 weeks of age and monitored for colitis symptoms. The results showed that CS delivered mice experienced an increased body weight loss and colon weight, together with higher colonic concentrations of TNF- $a$ and MPO compared with VD mice. Increased infiltration of inflammatory cells was present in CS delivered mice, as well as a downregulation in expression of the gut integrity genes occludin and tight junction protein 1 indicative of an impaired barrier function. The GM from CS delivered mice without colitis partly contributed to the increase in colitis symptoms when inoculated into germ-free recipient mice. In conclusion, CS increased sensitivity to oxazolone induced colitis in mice.
\end{abstract}

Mucosal Immunology (2019) 12:1348-1357; https://doi.org/10.1038/s41385-019-0207-8

\section{INTRODUCTION}

Cesarean section (CS) rates are increasing worldwide ${ }^{1}$ and in certain regions of China and Brazil, the use of CS as delivery mode has skyrocketed to $50-80 \%$ due to the massive use of elective CS for nonmedical reasons. ${ }^{2,3}$ Beyond risks of respiratory distress and breast feeding complications, epidemiological studies have suggested that the increasing trend of CS could be linked to the epidemic of chronic inflammatory diseases, including inflammatory bowel disease (IBD), in industrialized countries. ${ }^{4-6}$

The term IBD covers two gastrointestinal chronic inflammatory disorders. Crohn's disease, which consists of transmural inflammatory focuses that can affect any part of the gastrointestinal tract and ulcerative colitis, which is restricted to the colon with continuous inflammation and sometimes ulcers in the mucosa. ${ }^{7}$ The precise pathogenesis of IBD is still unclear, but a deregulated immune response against the gut microbiota (GM) in genetically susceptible patients seems to be a cornerstone of both Crohn's disease and ulcerative colitis. ${ }^{8,9}$ The significant role of the GM in disease etiology has also been demonstrated in murine models, in which fecal transfer of a colitis-prone GM induces colitis in the recipient mouse, ${ }^{10}$ and when the mice are kept under germ-free conditions, they fail to develop experimental colitis. ${ }^{11}$ Furthermore, probiotic treatment has shown to mitigate colitis symptoms by rescuing body weight loss, intestinal lymphocyte accumulation, and pro-inflammatory cytokine production in a T-cell induced colitis model. $^{12}$

Especially early in life, the GM is considered a key regulator of the pathogenesis of IBD. ${ }^{13}$ This has been demonstrated in an experimental model as re-colonization of neonatal germ-free mice, but not adult, prevented a severe degree of iNKT cellmediated oxazolone-induced colitis. ${ }^{14}$ Early colonization of the gut also initiates priming of the immune system with expansion and activation of intestinal regulatory T cells, ${ }^{15}$ which main function is to induce immune tolerance and dampen the excessive immune response that characterizes IBD. ${ }^{16}$ Regulatory T cells are decreased in the peripheral blood of IBD patients, ${ }^{17}$ and their role in IBD pathogenesis is most obvious in the adoptive transfer mouse model of colitis, in which colitis recovery was obtained after regulatory T-cell transfer. ${ }^{18}$

CS has been associated with a higher risk of ulcerative colitis and especially Crohn's disease., ${ }^{6,19}$ However, the oxazoloneinduced model, which is mainly a model for ulcerative colitis, was used in this study and the rationale for choosing this model was based upon earlier results from the CS mouse model in which a microbiota-regulated reduction of regulatory $T$ cells and an increased amount of iNKT cells were present in CS delivered mice. ${ }^{20}$ Since the disease expression of oxazolone-induced colitis is dependent upon GM and its stimulation of iNKT cells, ${ }^{14}$ this model was suitable to investigate the causality between CS and colitis development in mice.

The aim of this study was to investigate the impact of CS on experimental colitis by induction of oxazolone-induced colitis in mice delivered by CS and vaginal delivery (VD). We hypothesized that CS-delivered mice show aggravated colonic inflammation and gut permeability in the oxazolone-induced colitis mouse model due to a skewed microbial immune activation during birth. The isolated impact of the GM was investigated in germ-free mice inoculated with GM from conventional nontreated mice born by CS or VD.

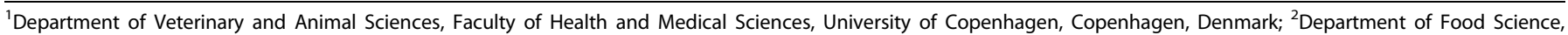

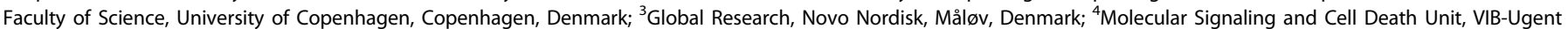

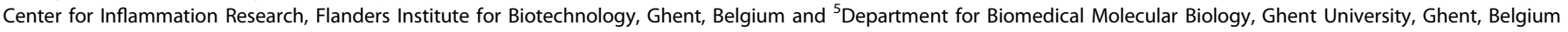
Correspondence: Line Fisker Zachariassen (lisfa@sund.ku.dk)

Received: 12 December 2018 Revised: 30 August 2019 Accepted: 7 September 2019

Published online: 25 September 2019 

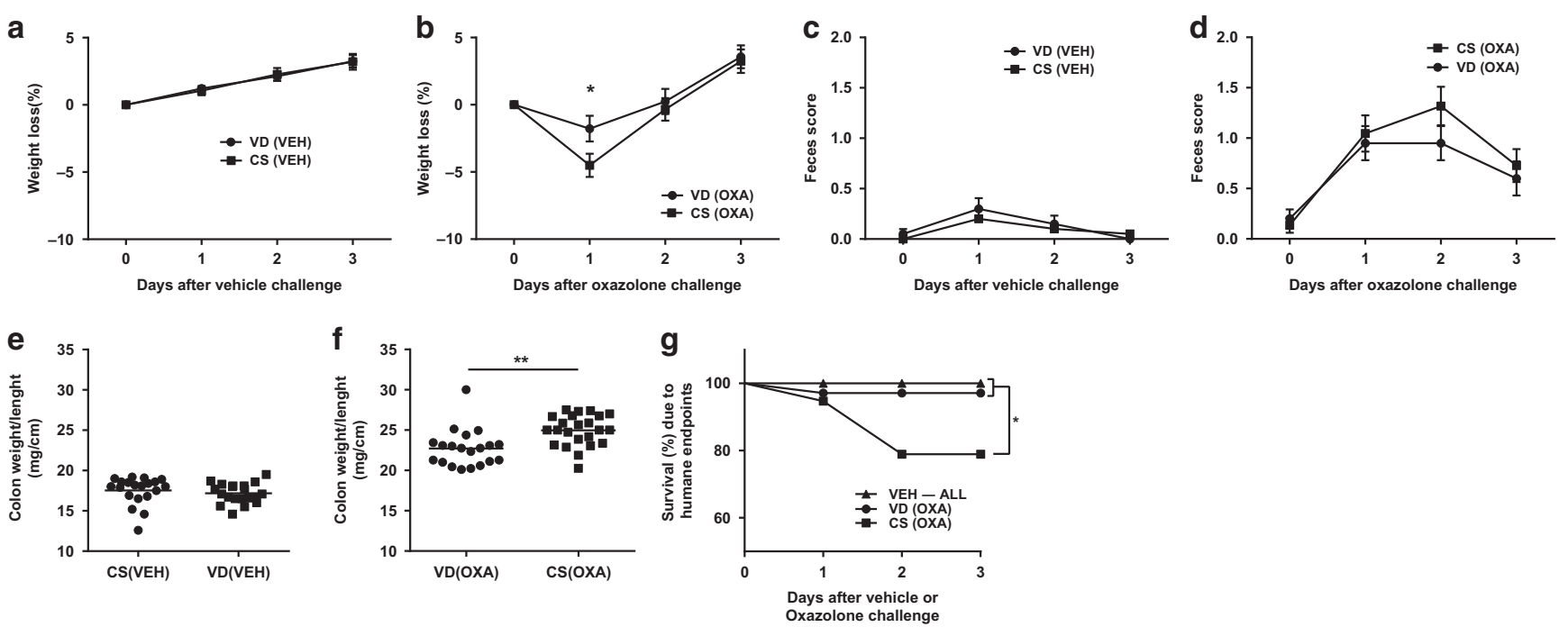

Fig. 1 Cesarean section increased disease symptoms in a murine model of experimental colitis. Plots illustrate symptoms of colitis in C57BL/6 mice ( 9 weeks) born by vaginal delivery (VD) or cesarean section (CS) with oxazolone-induced colitis (OXA) compared with vehicle treated (VEH) groups. Difference in weight loss (\%) calculated by two-way ANOVA 0-3 days after a vehicle treatment or b oxazolone challenge. n: VD $(\mathrm{VEH})=20, \mathrm{CS}(\mathrm{VEH})=20, \mathrm{VD}(\mathrm{OXA})=33, \mathrm{CS}(\mathrm{OXA})=30$. Difference in feces score calculated by two-way ANOVA $0-3$ days after c vehicle treatment or $\mathbf{d}$ oxazolone challenge. $0=$ hard and firm, $1=$ firm but sticky, $2=$ soft but still coherent, $3=$ soft with loss of shape or $4=$ liquid. $\mathrm{n}: \mathrm{VD}(\mathrm{VEH})=20, \mathrm{CS}(\mathrm{VEH})=20, \mathrm{VD}(\mathrm{OXA})=20, \mathrm{CS}(\mathrm{OXA})=22$. Difference in colon weight/length ratio calculated by student's $t$ test 3 days after e vehicle treatment or $\mathbf{f}$ oxazolone challenge. $n: \operatorname{VD}(\mathrm{VEH})=20, \mathrm{CS}(\mathrm{VEH})=20, \mathrm{VD}(\mathrm{OXA})=20, \mathrm{CS}(\mathrm{OXA})=22$. g Survival percentage of mice at the end of study, euthanized mice had a weight loss above $20 \%$, curve comparison calculated by Mantel-Cox test. $\mathrm{n}: \mathrm{VEH}(\mathrm{ALL})=40, \mathrm{VD}=39$, $\mathrm{CS}=38$. Bars represent mean and SEM. ${ }^{*} p<0.05,{ }^{* *} p<0.01$

\section{RESULTS}

Mice delivered by CS had more severe colitis symptoms characterized by increased weight loss and colon weight/length ratio compared with mice born by VD

All mice with induced colitis experienced a weight loss after oxazolone challenge while the vehicle groups gained weight after vehicle treatment (Fig. 1a, b). CS mice with colitis lost significantly more body weight day 1 after oxazolone challenge compared with VD colitis mice (Fig. 1b). No difference in initial weight in either the vehicle groups (VD(VEH): mean $=20.3 \mathrm{~g}, \quad S D=2.7 \mathrm{~g} . \quad \mathrm{CS}(\mathrm{VEH})$ : mean $=19.3 \mathrm{~g}, \mathrm{SD}=2.1 \mathrm{~g} . p(t$-test $)=0.4)$ or the oxazolone groups $(\mathrm{VD}(\mathrm{OXA}):$ mean $=18.5 \mathrm{~g}, \quad \mathrm{SD}=2.7 \mathrm{~g} . \quad \mathrm{CS}(\mathrm{OXA}):$ mean $=19.3 \mathrm{~g}$, $\mathrm{SD}=2.4 \mathrm{~g} . \quad p(t$-test $)=0.2$ ) was observed. Colitis induced mice, but not vehicle treated mice, showed softened fecal pellets day 1 after oxazolone challenge until euthanasia at day 3 (Fig. 1c, d). No difference in initial feces score was observed in either the vehicle groups (VD(VEHICLE): mean $=0.05 \mathrm{SD}=0.22$. $\quad \mathrm{CS}(\mathrm{VEHICLE})$ : mean $=0, S D=0 . p(t$-test $)=0.3$ ) or the oxazolone treated groups $(\mathrm{VD}(\mathrm{OXA}):$ mean $=0.19, \mathrm{SD}=4 . \mathrm{CS}(\mathrm{OXA}):$ mean $=0.14, \mathrm{SD}=0.4 . p$ $(t$-test $)=0.6)$. None of the mice had diarrhea and there was no significant difference in feces consistency between colitis mice born by CS and VD (Fig. 1d). All colitis-induced mice had a shortened colon with a higher organ weight compared with vehicle-treated mice (Fig. 1e, f). CS colitis mice presented with an increased colon weight/length ratio compared with VD delivered colitis mice (Fig. 1f).

Nine mice were euthanized at day 1 and 2 after oxazolone colitis challenge due to weight loss above $20 \%$ (Fig. 1g). A significantly larger proportion of CS delivered mice (8/38) experienced excessive weight loss after oxazolone challenge compared with VD mice (1/34). All animals euthanized due to humane endpoints were excluded from all other analysis

CS resulted in increased colonic infiltration of inflammatory cells and modifications in the gut integrity and permeability

The oxazolone-induced colitis was characterized by disseminated colonic infiltration of mononuclear cells and neutrophil granulocytes compared with the vehicle treated groups (Fig. 2a $\pm f$ ). Likewise, cell turnover defined by the number of mitotic figures in the colon epithelium was increased in mice induced with colitis (Fig. $2 \mathrm{~g}, \mathrm{~h}$ ). Oxazolone treated CS delivered mice had increased infiltration of mononuclear cells and neutrophil granolucytes, together with a higher cell turn over compared with oxazolonetreated mice born by VD (Fig. 2f, h).

Oxazolone treated mice born by CS showed a tendency $(p=0.06)$ to absorb more FITC labeled dextran across the gut epithelium compared with oxazolone treated VD mice. No difference in the concentration of absorbed FITC labeled dextran was present in the vehicle groups (Fig. 2i, j). The concentration of absorbed FITC labeled dextran seems to be higher in the vehicle treated groups, however, this is most likely due to batch variation of FITC labeled dextran.

Although the expression of the mucus producing Mucin-1 and Mucin-2 genes seem to be increased in oxazolone treated mice, there was no difference in either vehicle or oxazolone treated groups according to delivery mode (Fig. 2k, I). The expression of Tight junction protein 1 was slightly increased in the vehicle treated CS delivered mice compared with vehicle VD mice (Fig. $2 \mathrm{k}$ ). This difference was reversed in the oxazolone-induced colitis mice in which both the gut integrity genes Occludin and Tight junction protein 1 was downregulated compared with the colitis mice born by VD (Fig. 2l). There was no difference between the oxazolone treated groups in the amount of bacterial originating 16S rRNA gene copies that have crossed the gut barrier to the MLN (Data not shown).

Colonic concentrations of TNF- $a$ and MPO were increased in CS delivered mice with oxazolone-induced colitis, while colonic gene expression of Tgf $\beta, C x C l 1, T / r 2$, and TI1 a was downregulated Vehicle treated mice delivered by CS had higher concentrations of TNF-a in the colon compared with VD mice (Fig. 3a). No differences in serum cytokine concentration were present in the vehicle groups regardless of delivery mode (Fig. 3b). The amount of IL-2, IL-4, and IL-12p70 in colon and serum together 


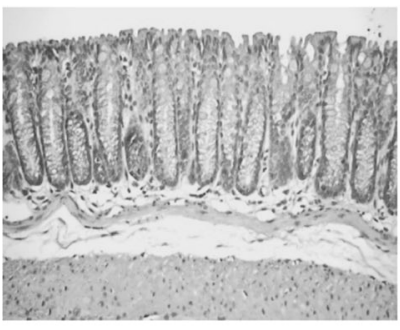

b

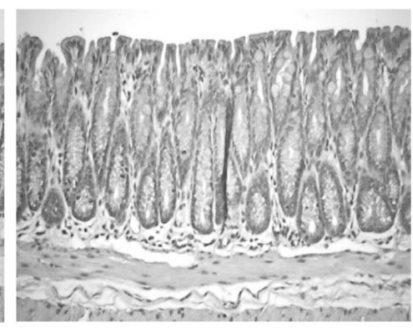

C

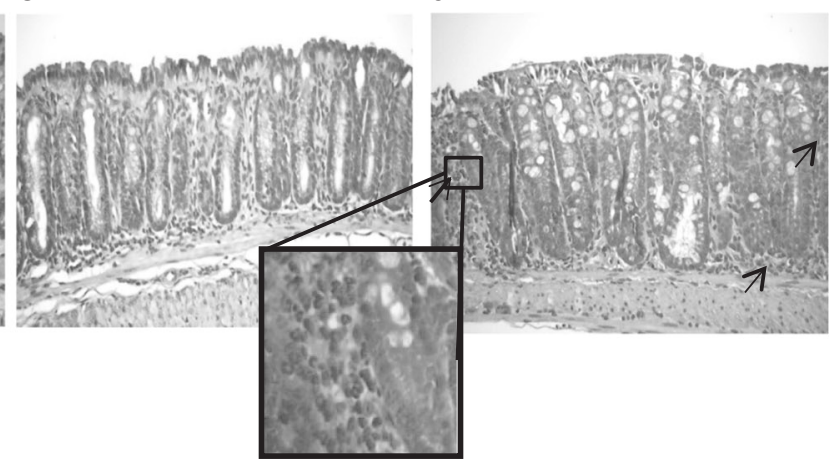

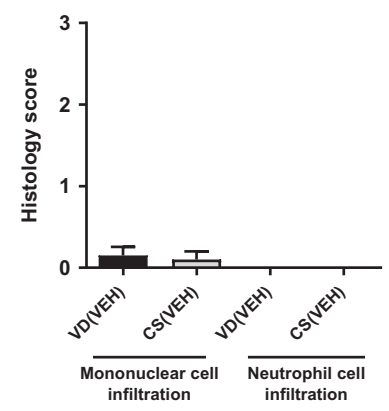

i

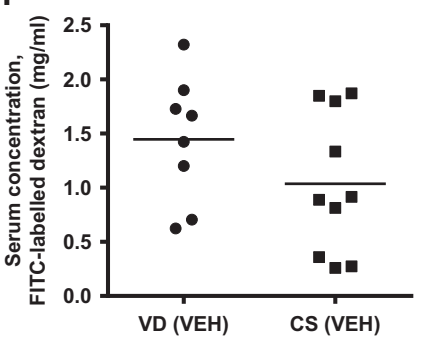

f

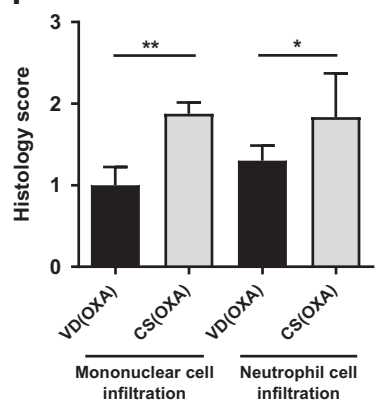

j

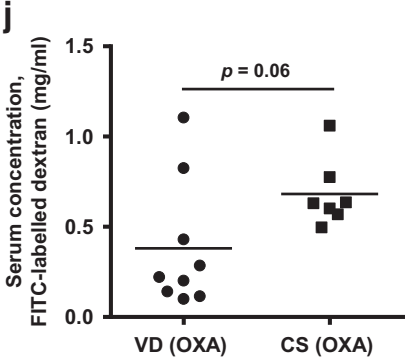

g
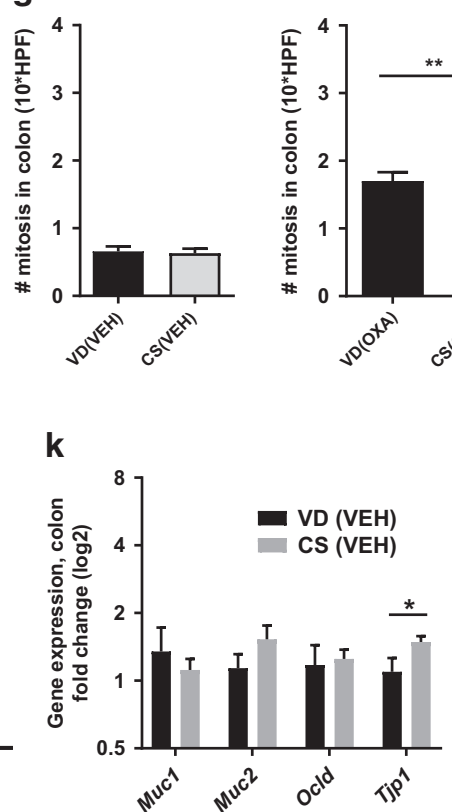

h

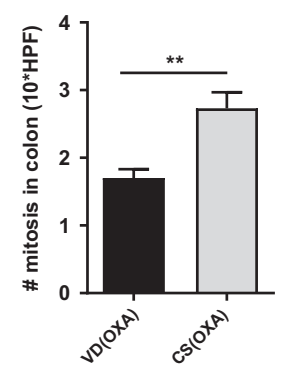

I

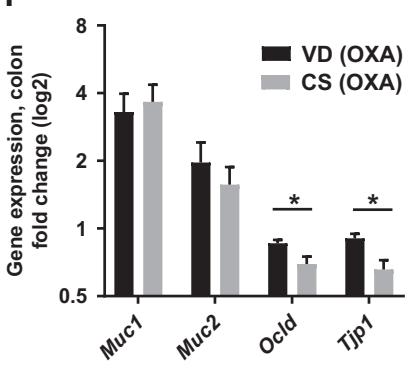

Fig. 2 Cesarean section increased colon infiltration of inflammatory cells and gut permeability. Plots illustrate gut integrity results from C57BL/6 mice ( 9 weeks) born by vaginal delivery (VD) or cesarean section (CS) with oxazolone-induced colitis (OXA) compared with vehicle (VEH) groups. Hematoxylin and eosin staining of colon with a no inflammatory cell infiltration b mild infiltration of inflammatory cells, c moderate infiltration of inflammatory cells d severe infiltration of inflammatory cells, arrows = neutrophil granulocytes. Mean score of mononuclear cells and neutrophil granulocytes in colon 3 days after $\mathbf{e}$ vehicle treatment or $\mathbf{f}$ oxazolone challenge. $0=$ no infiltration $1=$ mild infiltration 2 =moderate infiltration and $3=$ severe infiltration. $n \geq 10$. Cell turnover in colon epithelium characterized by mean number of mitotic figures counted in $10^{*}$ high power fields (40X)(HPF) 3 days after $\mathbf{g}$ vehicle treatment or $\mathbf{h}$ oxazolone challenge. $n \geq 10$. Concentration of FITC labeled dextran $(\mathrm{mg} / \mathrm{ml})$ passed through the gut barrier to the bloodstream $2 \mathrm{~h}$ after oral gavage at day 3 after $\mathbf{i}$ vehicle treatment or j oxazolone challenge. $n \geq 7$. Gene expression measured by qPCR 3 days after $\mathbf{k}$ vehicle treatment or I oxazolone challenge. Data are presented as log2 fold change of colonic integrity genes Mucin-1 (Muc1), Mucin-2 (Muc2), Occludin (Ocld), and Tight junction protein 1 (Tjp1) $n \geq 10$. Differences were calculated by student's $t$ test for parametric data and Mann Whitney's U-test for nonparametric data. Bars represent mean and SEM. ${ }^{*} p<0.05,{ }^{* *} p<0.01$

with IFN- $\gamma$ in serum were below the detection range of the assay in the vehicle treated groups and therefore not included in the results (Fig. 3a, b). Challenge with oxazolone increased the concentration of the majority of measured cytokines in colon and serum. Colitis-induced mice delivered by CS still had a significantly higher concentration of colonic TNF-a (Fig. 3c), but also a higher serum concentration of IL- $1 \beta$ was present in colitisinduced mice delivered by CS compared with VD mice (Fig. $3 \mathrm{~d}$ ). The concentration of the neutrophil granulocyte-associated enzyme myeloperoxidase (MPO) was significantly higher in colon and serum from colitis-induced mice delivered by CS compared with VD colitis mice. No difference in MPO concentration was present in the vehicle groups regardless of delivery mode (Fig. 3e, f).
Vehicle treated mice delivered by CS had an upregulated gene expression of $\mathrm{CxCl} 1$ and Tnfa in the colon compared with VD vehicle treated mice (Fig. $3 \mathrm{~g}$ ). The gene expression of $\mathrm{CxCl} 1$ was reversed after oxazolone treatment in the CS delivered mice which presented with a downregulated expression of colonic Cxcl1 together with Tgf $\beta, T / 1 a$, and T/r2 compared with the VD colitis mice (Fig. 3h).

Delivery by CS reduced the proportion of regulatory $\mathrm{T}$ cells and increased the initial proportion of iNKT cells

CS mice, both vehicle and oxazolone treated, showed a reduced proportion of $\mathrm{CD}_{103}{ }^{+}$and $\mathrm{CD}^{+} 9^{+}$activated $\mathrm{FoxP}^{+} \mathrm{CD}^{+}$ regulatory $T$ cells in the mesenteric lymph node (MLN) and spleen compared with VD mice (Fig. 4a-f). No significant differences were 
a

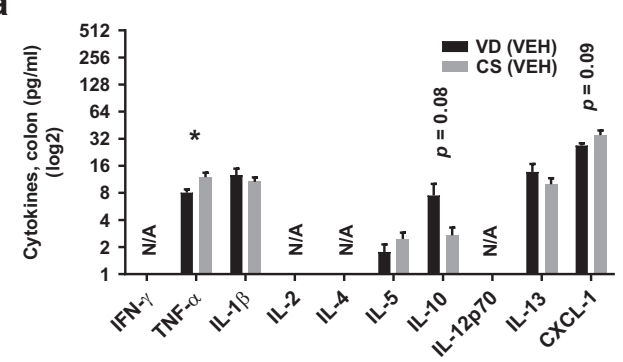

C

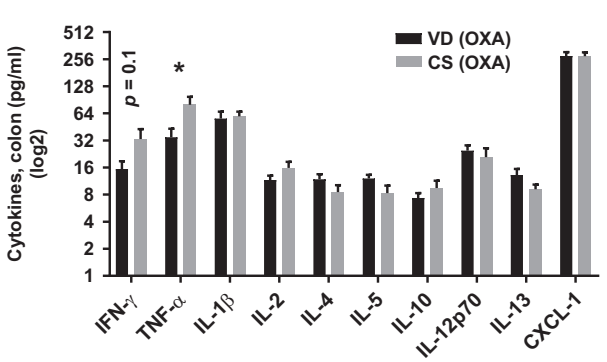

$\mathbf{e}$

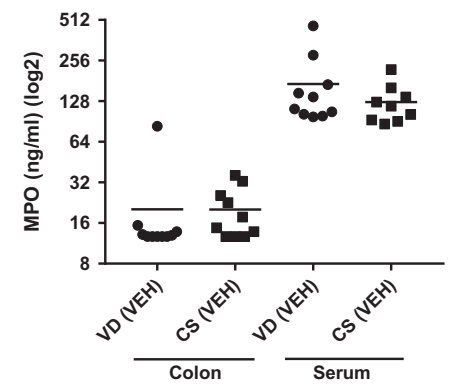

g

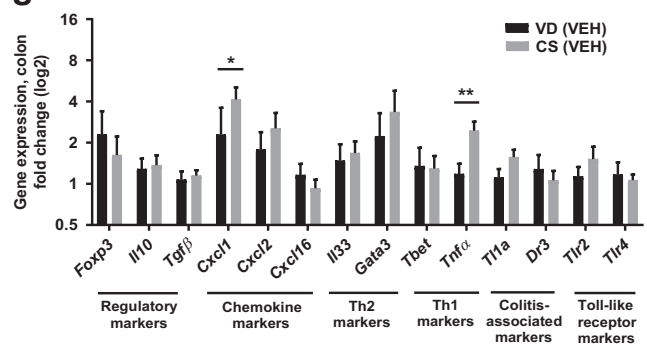

b

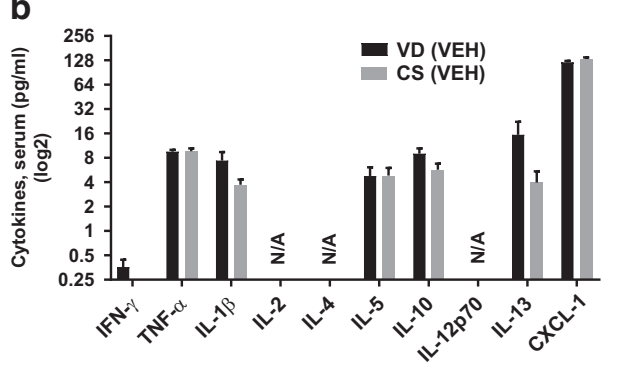

d

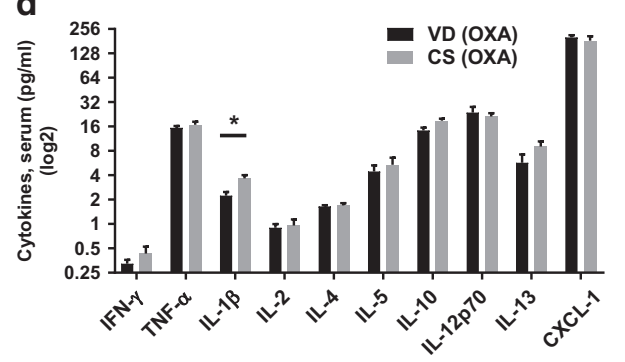

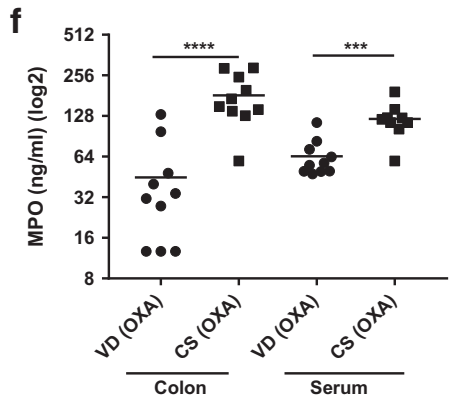

h

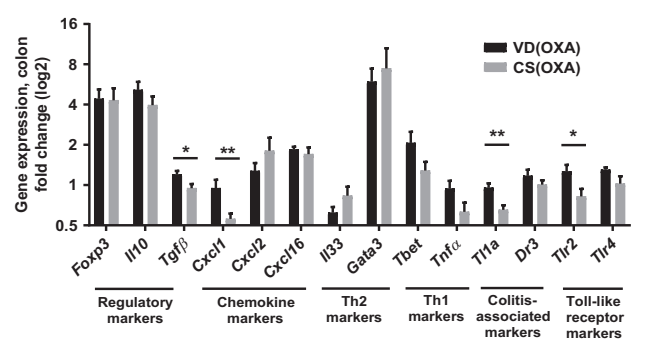

Fig. 3 Cesarean section increased inflammatory markers in a murine model of experimental colitis. Plots illustrate the degree of inflammation in C57BL/6 mice (9 weeks) born by vaginal delivery (VD) or cesarean section (CS) with oxazolone-induced colitis (OXA) compared with vehicle treated (VEH) groups. a-d Multiplex assay and ELISA results of $\mathbf{a}, \mathbf{c}$ colonic ( $100 \mathrm{mg}$ tissue) and $\mathbf{b}$, $\mathbf{d}$ serum cytokine concentrations obtained 3 days after $\mathbf{a}, \mathbf{b}$ vehicle treatment or $\mathbf{c}, \mathbf{d}$ oxazolone challenge. Concentrations are plotted on a logarithmic scale in pg/ml. $n \geq 10$. ELISA results of colonic (100 mg tissue) and serum cytokine concentration of myeloperoxidase (MPO) obtained 3 days after e vehicle treatment or $\mathbf{f}$ oxazolone challenge. Concentrations are plotted on a logarithmic scale in $\mathrm{ng} / \mathrm{ml} . n=10$. Logarithmic fold change of colonic gene expression measured by qPCR 3 days after $\mathbf{g}$ vehicle treatment or $\mathbf{h}$ oxazolone challenge. $n \geq 10$. Differences were calculated by student's $t$ test for parametric data and Mann-Whitney's U-test for nonparametric data. Bars represent mean and SEM. ${ }^{*} p<0.05,{ }^{* *} p<0.01,{ }^{* * *} p<0.001$, $* * * * p<0.0001$

observed in the percentages of $\mathrm{CD}^{+}{ }^{+}$cells or the FoxP3 ${ }^{+} \mathrm{CD} 4^{+}$ $T$ cells (Data not shown). A higher proportion of iNKT cells was present in colonic intraepithelial lymphocytes (IEL) in vehicle CS delivered mice compared with vehicle VD mice (Fig. 4g). The same difference was not present in the oxazolone-treated mice where all mice had an equally high level of iNKT cells in spleen and colon after oxazolone challenge (Fig. 4h).

The distribution of NKG2D activated $\mathrm{CD}^{+}$and $\mathrm{CD} 8 \mathrm{a} \beta^{+}$T cells was measured in the spleen, colonic IEL, and lamina propria lymphocytes (LPL) in the oxazolone-treated groups, but there were no differences in these cell subsets according to delivery mode (data not shown).

GM from CS mice was partly responsible for the disease severity and the inflammatory response in oxazolone-induced colitis mice To investigate if inoculation of GM obtained from mice born by CS or VD was sufficient to affect disease sensitivity, disease parameters which significantly differed in conventional mice of different delivery mode, were measured in the inoculated recipient mice. 

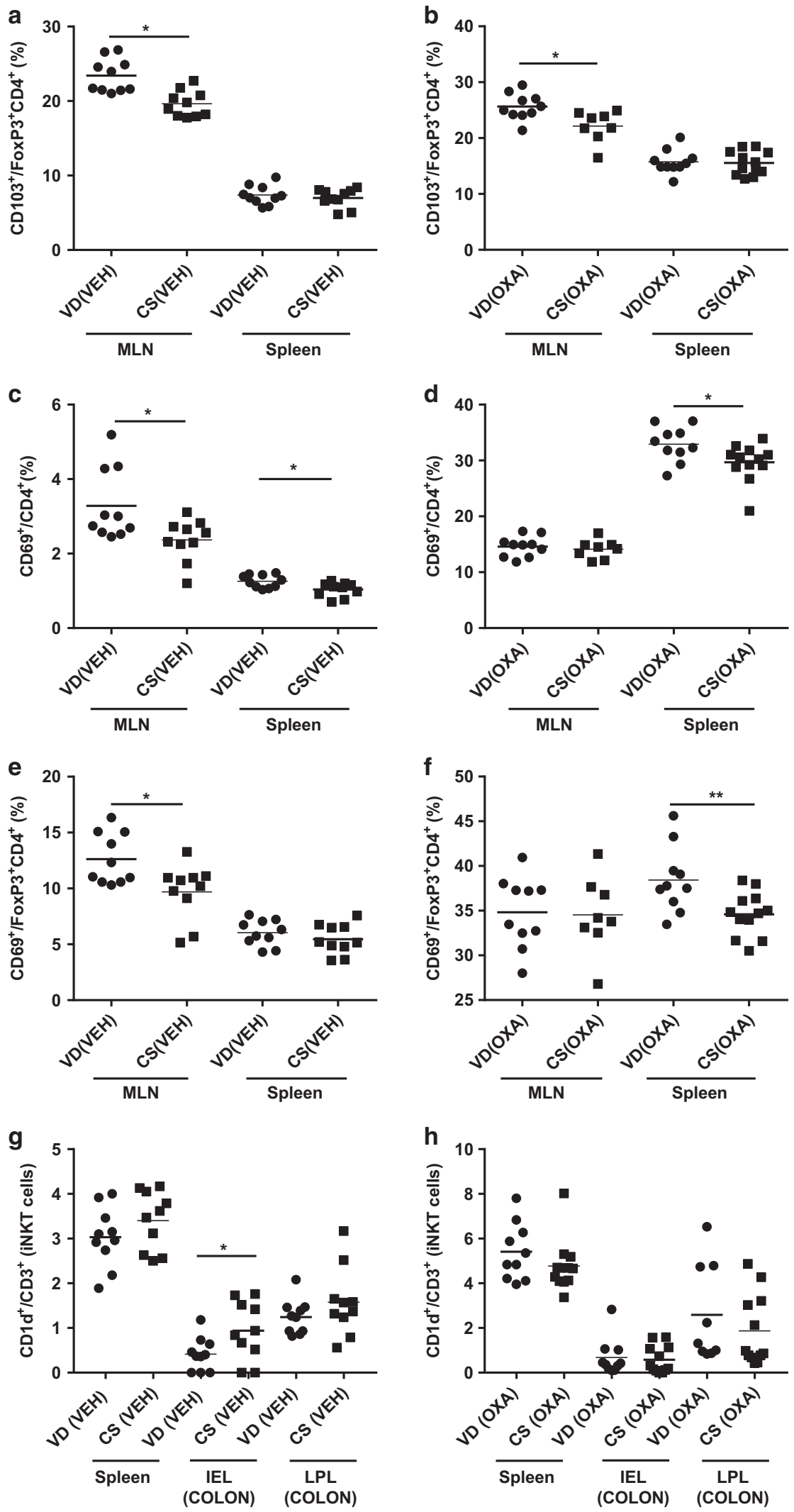

Fig. 4 Cesarean section in mice reduced the proportion of regulatory $\mathrm{T}$ cells and increased the initial proportion of invariant natural killer T (iNKT) cells. Plot illustrates flow cytometry results of regulatory T cells and iNKT from C57BL/6 mice (9 weeks) born by vaginal delivery (VD) or cesarean section (CS) with oxazolone induced colitis (OXA) compared with vehicle groups (VEH). a-f Regulatory T cells isolated from the mesenteric lymph node (MLN) and spleen. $\mathbf{g}$, $\mathbf{h}$ iNKT cells isolated from the spleen, colonic intraepithelial lymphocytes (IEL) and colonic lamina propria lymphocytes (LPL). a, b Percentages of $\mathrm{CD}_{103}{ }^{+}$regulatory Foxp $3^{+} \mathrm{CD} 4^{+} \mathrm{T}$ cells. c, d Percentages of $\mathrm{CD} 69^{+} \mathrm{CD} 4^{+} \mathrm{T}$ cells. e, $\mathbf{f}$ Percentages of $\mathrm{CD} 9^{+}$regulatory FoxP3 ${ }^{+} \mathrm{CD} 4^{+} \mathrm{T}$ cells. Significance calculated with student's $t$ test. Error bars represent SEM. $n=10,{ }^{*} p<0.05,{ }^{*} p<0.01$ 

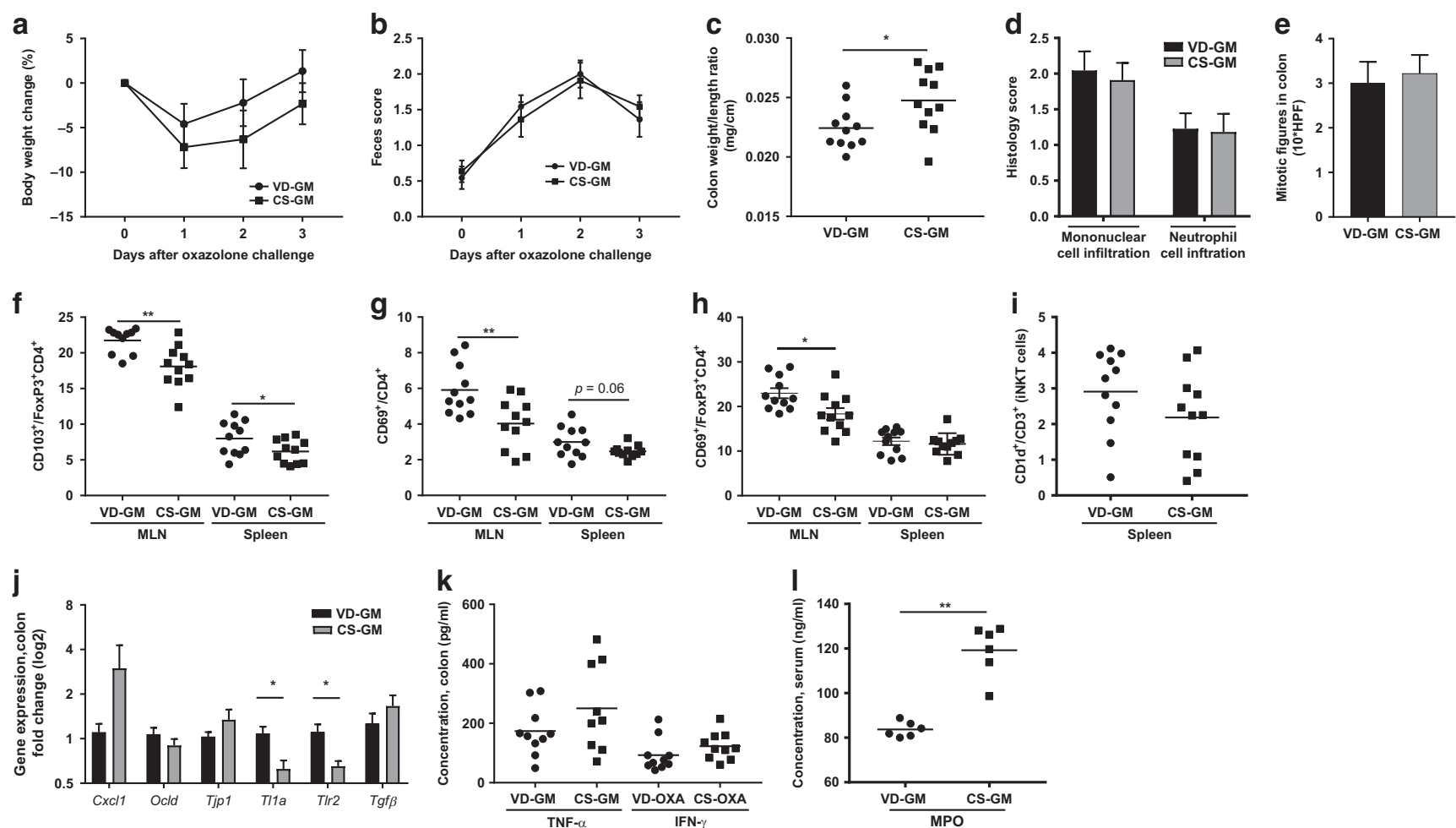

Fig. $5 \mathrm{GM}$ in CS mice was partly responsible for the disease severity and the inflammatory response in oxazolone-induced colitis. Plots illustrate results from germ-free C57BL/6 mice ( 9 weeks) with oxazolone induced colitis (OXA) inoculated with gut microbiota (GM) obtained from conventional mice born by vaginal delivery (VD) or cesarean section (CS). a Weight loss (\%) $0-3$ days after oxazolone challenge. $n=11$. b Feces score 0-3 days after oxazolone challenge, $0=$ hard and firm, $1=$ firm but sticky, $2=$ soft but still coherent, $3=$ soft with loss of shape or $4=$ liquid. $n=11$. c Colon weight/length ratio 3 days after oxazolone challenge. $n=11 \mathrm{~d}$ Mean score of mononuclear cells and neutrophils in colon, $0=$ no infiltration $1=$ mild infiltration $2=$ moderate infiltration and $3=$ severe infiltration. $n=11$. e Cell turnover in colon epithelium characterized by mean number of mitosis counted in 10*high power fields (40X)(HPF). $n=11 \mathbf{f}-\mathbf{h}$ Flow cytometry results of regulatory T cells isolated from the mesenteric lymph node $(\mathrm{MLN})$ and spleen. $n=11$. f Percentages of $\mathrm{CD} 103^{+}$regulatory Foxp $3^{+} \mathrm{CD} 4^{+} \mathrm{T}$ cells. g Percentages of $\mathrm{CD} 69^{+} \mathrm{CD} 4^{+} \mathrm{T}$ cells. $\mathbf{h}$ Percentages of $\mathrm{CD} 69^{+}$regulatory FoxP3 ${ }^{+} \mathrm{CD} 4^{+} \mathrm{T}$ cells. i Percentages of $\mathrm{CD} 1 \mathrm{~d}^{+} \mathrm{CD} 3^{+}$restricted invariant natural killer T cells (iNKT) isolated from the spleen. $\mathbf{j}$ Colonic gene expression measured by qPCR and presented as logarithmic fold change, $n=11$. k Concentration of TNF- $\alpha$ and IFN- $\gamma$ in $100 \mathrm{mg}$ colon tissue measured by ELISA. $n=10$. I Concentration of serum myeloperoxidase (MPO) measured by ELISA. $n=6$. Differences was calculated with students $t$ test for parametric data and Mann-Whitney test for nonparametric data. Bars represent mean and SEM. ${ }^{*} p<0.05,{ }^{* *} p<0.01$

$16 \mathrm{~s}$ sequencing of GM from the inoculated mice showed a similar GM as the respective donors. Despite significant difference in abundance of several taxa including Prevotella spp. between mice inoculated with GM from conventional VD mice (VD-GM) or CS-delivered mice (CS-GM) the overall clustering of the GM was comparable (Supplementary Fig. 1A-D).

All inoculated mice experienced a weight loss and softened feces after oxazolone challenge, but there were no differences between CS-GM and VD-GM inoculated mice (Fig. 5a, b). The colon weight/length ratio was significantly higher in CS-GM inoculated mice compared with VD-GM inoculated mice (Fig. 5c). Only one mouse from the CS group had to be euthanized before the end of the study due to a weight loss above 20\% (Data not shown).

All inoculated mice with colitis showed infiltration of mononuclear cells and neutrophils, together with increased cell turnover resembling that of the conventional colitis mice (Fig. 5d, e). However, no differences in the degree of cell infiltration or cell turnover were observed in the inoculated mice according to delivery mode (Fig. $5 d$, e).

Mice inoculated with CS-GM had the same reduction as their $\mathrm{GM}$ donors in $\mathrm{CD}_{103}{ }^{+}$and $\mathrm{CD} 9^{+}$activated $\mathrm{FoxP}^{+} \mathrm{CD}^{+}$ regulatory $T$ cells in MLN and spleen compared with VD-GM inoculated mice (Fig. $5 f-h$ ). No differences were observed in the percentages of $\mathrm{CD}^{+}{ }^{+} \mathrm{T}$ cells or the FoxP3 ${ }^{+} \mathrm{CD}^{+}{ }^{+} \mathrm{T}$ cells (data not shown). The proportion of $\mathrm{CD}_{1} \mathrm{~d}^{+} / \mathrm{CD}^{+}$restricted iNKT cells in the spleen was similar in all inoculated mice (Fig. 5i).
Similar to the conventional colitis mice, gene expression of Tl1a and T/r2 was downregulated in colitis-induced mice inoculated with CS-GM. No difference was observed in the gut integrity genes (Fig. 5j) or in the colonic cytokine concentration of TNF- $a$ and IFN$\gamma$ (Fig. 5k). Serum MPO concentration was increased in mice inoculated with CS-GM compared with VD-GM (Fig. 5I) as in the conventional mice.

\section{DISCUSSION}

The association between delivery mode and risk of IBD is poorly understood and requires long term human follow-up studies, which are challenging due to a complex multifactorial pathogenesis with many confounders such as the use of antibiotic during CS and childhood, ${ }^{21}$ breast feeding vs. formula feeding, ${ }^{22}$ and preterm birth. ${ }^{23}$ To better understand causality and investigate CS regulated disease mechanisms involved in the pathogenesis, a good and valid animal model combining CS with experimental colitis is needed.

This study shows, in a previously established CS murine model, that CS aggravates inflammation in the oxazolone-induced colitis mouse model. Mice delivered by CS had increased weight loss and colon weight/length ratio suggesting a higher degree of colonic inflammation compared with VD born mice. Excessive weight loss resulting in euthanasia before the end of the study was also more pronounced in the CS delivered mice. The colonic inflammation 
was characterized by massive infiltration of inflammatory cells and a higher rate of cell turnover in CS-delivered mice compared with mice born by VD. This was also confirmed by higher colonic concentration of the pro-inflammatory cytokines TNF- $a$ and MPO and systemic concentrations of IL-1 $\beta$ and MPO. There was a strong indication of reduced gut barrier integrity in the colitis-induced mice born by CS compared with VD mice. Disruption of the gut integrity allows luminal content to pass and trigger the intestinal immune response. ${ }^{24}$ IBD comprises an impaired gut barrier characterized by downregulation of the tight junction proteins occludin and claudin, ${ }^{25}$ but also presents with high concentrations of TNF-a, which may alter the gut barrier. ${ }^{26}$

Tnfa gene expression was indeed increased in CS vehicletreated mice compared with VD vehicle-treated mice in line with the higher cytokine concentration of TNF-a. The TNF-a concentration was also increased in oxazolone-treated mice delivered by CS compared with VD mice and may as such play a significant role in the impaired gut integrity and aggravated disease symptoms evident in CS colitis-induced mice. On the contrary, Tnfa gene expression was not significantly different in the CS colitis-induced mice compared with the VD colitis-induced mice, which may have been a result of a negative feedback on transcription following a prolonged high TNF- $a$ concentration. In addition, transcription and translation of cytokines are not always correlated due to differences in half-life, degradation time, and stability. ${ }^{27}$

The vehicle-treated CS mice had an increased gene expression and colonic protein concentration of the neutrophil chemokine CXCL-1 which was not seen in the colitis-induced mice. In fact, the expression of $\mathrm{CxCl} 1$ was downregulated in the CS mice compared with VD mice which seems contradictive to the increased colonic infiltration of neutrophils and the higher concentration of colonic MPO found in CS colitis-induced mice compared with VD colitisinduced mice. A possible explanation for this could be the nature of the markers and the time of inflammation the markers has been measured. CXCL-1 is involved in the recruitment of neutrophils to the inflammation site, ${ }^{28}$ whereas MPO is excreted from activated neutrophils. ${ }^{29}$ Colon samples are obtained three days after challenge where clinical weight and fecal score of the mice have already started to normalize. At this time point, an increased infiltration and thereby excretion of MPO would be expected, while the need for recruiting new neutrophils has likely diminished.

Mice delivered by CS showed a decrease in T/r2 gene expression which is interesting in light of the fact that recognition of intestinal bacterial epitopes by TLR2 has proven to be central for gut homeostasis and gut integrity in TLR2 ${ }^{-1-}$ mice. ${ }^{30}$ Gene expression of Tnfsf15 (TL1a) was also downregulated in mice delivered by CS. Elevated mRNA expression of TI1a and its receptor Tnfsf25 (Dr3) are usually associated with colitis severity, ${ }^{31}$ but a recent study suggests a protective role of the TL1a/DR3 axis in the DSS induced murine model, since $\mathrm{TI}_{1} \mathrm{a}^{-1-}$ and $\mathrm{Dr}^{-1-}$ mice presented with a higher weight loss, increased colonic infiltration of inflammatory cells, and severe epithelial injury. This aggravation of colitis symptoms was probably due to a reduction of $\mathrm{CD}^{+} \mathrm{CD}^{+} 5^{+} \mathrm{FoxP}^{+}$regulatory $\mathrm{T}$ cells in $\mathrm{MLN}$ in $\mathrm{Tl}^{-1} \mathrm{a}^{-1-}$ and $\mathrm{Dr}^{-1-}$ mice. ${ }^{32}$ These results are in alignment with this study in which CS delivered mice had lower gene expression of TI1a together with a reduced percentage of activated regulatory $T$ cells in MLN and spleen.

Regulatory T cells are crucial in keeping immune balance ${ }^{17}$ and a dysregulation of this cell subset has been reported as a major player in the pathogenesis of IBD. ${ }^{33}$ Particularly $\mathrm{CD}_{103}{ }^{+}$and $\mathrm{CD}^{+} 9^{+}$regulatory $\mathrm{T}$ cells have proven to be highly suppressive with an alleviating effect on inflammatory diseases ${ }^{34,35}$ which is in line with the reduced percentage of exactly these cells in CSdelivered mice. iNKT cells have also been shown to play a causative role in oxazolone-induced colitis. ${ }^{14}$ We have previously shown that CS is associated with an increased proportion of splenic iNKT cells, ${ }^{20}$ and in this study we find an increase in iNKT cells in colonic IELs. The increased amount of iNKT cells in the vehicle treated CS mice could very likely contribute to the severity of the iNKT cell-mediated oxazolone colitis symptoms. There was a notable rise in the overall percentage of iNKT cells in all oxazolone-induced mice, which has probably diminished the group differences observed in vehicle-treated groups.

A well-balanced GM is crucial for anatomy and normal function of the gut barrier. ${ }^{30}$ The protective role of the GM in intestinal immune homeostasis and barrier integrity has been verified in antibiotic treated mice, which showed severe morbidity and mortality when challenged with the mucosal irritative dextran sodium sulfate. ${ }^{30}$ Altered GM composition in CS delivered mice has previously been noted ${ }^{36,37}$ and our recent data showed a higher abundance of especially Prevotella spp. in CS delivered mice. ${ }^{20}$ Prevotella spp. has previously been classified as colitogenic by increasing the level of Th17 cells and neutrophils in IBD patients, $^{38}$ and colonization with a Prevotella-rich GM also increased susceptibility to experimental colitis in mice. ${ }^{39}$ Transferring GM from Prevotella-rich CS delivered donors showed that the immunologic phenotype was transferable with the CS-GM, while colitis symptoms, except the colon weight/length ratio and MPO concentration, remained comparable between the CS-GM and VDGM inoculated mice. It is important to note, that even though the CS-GM recipients acquired the same increased abundance of the colitogenic bacteria Prevotella spp. as the donor mice, the overall clustering of the GM had changed during inoculation so CS-GM and VD-GM clustered together. This could have reduced the effect of the CS altered GM on colitis symptoms.

Regardless, the reduced level of activated regulatory $T$ cells as well as the reduced gene expression of TIr2 and TI1a were transferable with the GM as we have also demonstrated previously. ${ }^{20}$ Transfer of the reduced level of activated regulatory T cells with the CS-GM could be a direct effect of the decreased TIr2 and TI1a expression in both donor and recipient mice, since both TLRs and TL1a signaling are involved in the activation of regulatory $T$ cells as mentioned above, ${ }^{32}$ however, in light of the marginal effect on colitis sensitivity it suggests that other immunological parameters are necessary to aggravate the colitis response to oxazolone observed in CS mice such as e.g., the high CXCL-1 and TNF-a concentrations associated with CS delivery which were non-transferable.

IBD is considered a multifactorial disease in which genetics, immune responses, and environmental factors all contribute to disease pathogenesis. ${ }^{7}$ It is, therefore, not unlikely that other factors regulated by delivery mode could affect colitis sensitivity in parallel with the GM. Preterm birth has been associated with increased risk of IBD in humans and even though the surgical procedure in mice is conducted at day 21 of pregnancy to ensure full term mouse pups, our own recent results showed that many of the pups still have a slightly lower birth weight compared with VD mouse pups, ${ }^{20}$ which should not be disregarded as a possible confounding factor. Physical stress and hormonal exposure during birth and in the neonatal period are also factors which are reliant on delivery mode ${ }^{40,41}$ and have shown to affect the immune system of the neonate, ${ }^{42-44}$ but their long term effects on IBD development remains unclear.

In conclusion, CS increased sensitivity to oxazolone-induced colitis characterized by increased weight loss, organ weight, and intense mononuclear and neutrophil cell infiltration as well as increased pro-inflammatory cytokines and reduced gut barrier integrity, which are all symptoms that characterize human IBD. ${ }^{7}$ Several immunological features were transferable with the GM, but the colitis phenotype was GM independent. Instead, the vehicle-treated mice demonstrated that CS give rise to an intestinal low grade inflammation with high concentration of CXCL-1 and TNF- $a$, which likely could be the causative trigger of an impaired barrier function and aggravated inflammatory 
response when challenged with oxazolone. TNF-a blockers are already widely used as therapeutic intervention ${ }^{45}$ as it is over secreted by innate cells in lamina propria of patients with IBD. ${ }^{46}$ This is the first study that links delivery mode to disease development and demonstrates the suitability of a CS delivered oxazolone-induced colitis model for further investigations of the mechanisms underlying perinatal health in CS delivered infants in risk of developing IBD.

\section{MATERIALS AND METHODS}

See Supplementary material for a detailed description of ethics, materials and methods.

Animals, breeding, and CS

Conventional and germ-free C57BL/6NTac mice (Taconic, Lille Skensved, DK/Germantown, NY) were housed in our AAALAC accredited barrier protected and germ-free facility (Faculty of Health and Medical Sciences, University of Copenhagen, Frederiksberg, Denmark) in open cages with ad libitum access to Altromin 1324 diet (Brogaarden, Lynge, Denmark) and tap water. Germ-free animals were housed in HEPA-ventilated isolators (PFI systems, Milton Keynes, UK) and given sterile diet and water.

Dams were time-mated for $48 \mathrm{~h}$ and separated into foster, CS, and VD dams. At day 20 of pregnancy, CS was performed by hysterectomy and the pups were transferred to a foster mom within $30 \mathrm{~min}$. Likewise, VD pups were transferred to a foster mom at day 0 . A minimum of three litters were used in each group and no litter differences were observed. The total number of mice in the conventional study; Oxazolone treated groups: $\operatorname{VD}(O X A)=39$, $\mathrm{CS}(\mathrm{OXA})=38$, and vehicle treated groups: $\mathrm{VD}(\mathrm{VEH})=20$, $\mathrm{CS}(\mathrm{VEH})=20$. To overcome tissue limitation, the mice were split into smaller groups for each colon analysis. The total number of mice in the inoculation study; VD-GM $=10, C S-G M=10$.

\section{Germ-free status and fecal inoculation}

Germ-free status was confirmed before fecal transfer both by fecal culturing and PCR methods as described previously. ${ }^{20}$ For inoculation, feces from conventional mice without colitis born by CS or VD were dissolved in sterile PBS and inoculated into germ-free mice. See Supplementary material for detailed description of germ-free testing and fecal inoculation procedures.

Oxazolone-induced colitis

All oxazolone-induced mice (8 weeks) were sensitized on day 1 and 4 with $150 \mu \mathrm{l} 3 \%(\mathrm{w} / \mathrm{v}$ ) oxazolone (Sigma-Aldrich, St. Louis, MO) dissolved 5:1 in ethanol:oil on the shaved abdomen. At day 7, all mice were intra-rectally challenged with $1 \%(\mathrm{w} / \mathrm{v})$ oxazolone dissolved in $50 \%$ ethanol under anesthesia with $2 \%$ isoflurane. The vehicle groups were given the vehicle only. The inoculated mice were briefly located outside the isolator during intrarectal oxazolone challenge. Here, the bacterial load was minimized by transferring the mice in closed cages and procedures performed under aseptic conditions. Mice were euthanized at day 10 after first oxazolone sensitization.

Colitis symptoms and post mortem analysis

Colitis status was monitored by weight change and fecal consistency at day $0,7,8,9$, and 10 of the experiment. Fecal consistency was evaluated by a scoring system used previously, ${ }^{47}$ modified from Murthy et al. $0=$ hard and firm, $1=$ firm but sticky, $2=$ soft but still coherent, $3=$ soft with loss of shape or $4=$ liquid. After euthanasia, the length of the colon was measured from the caeco-colic junction to anus, rinsed with $0.3 \mathrm{ml} \mathrm{PBS}$ and weighed. The proximal $1 / 3$ of the colon was discarded and the remaining part was cut longitudinally in halves for further analysis.
FITC-labeled dextran

To evaluate the gut barrier integrity, mice were fasted for four hours before oral dosing of FITC-labeled dextran (4 kDa, Sigmaaldrich) at day 10 of the experiment. After two hours, orbital blood was collected and the concentration of serum FITC-labeled dextran was measured by spectrophotofluorometry. See Supplementary material for a detailed protocol.

High throughput sequencing of the GM from inoculated mice Feces were obtained from inoculated mice before oxazolone treatment (8 weeks). DNA extraction, PCR amplification of the V3 region, normalization of the pooled library, sequencing using Illumina NextSeq (Illumina), and data analysis were carried out as previously described. ${ }^{20}$

Real time PCR ( $q P C R$ ) of bacterial epitopes in the mesenteric lymph node (MLN)

DNA was extracted from MLN of conventional mice using DNeasy Blood and Tissue Kit (Qiagen, Hilden, Germany) according to manufacturer's protocol. An initial bead beading step for $2 \times 45 \mathrm{~s}$ (FastPrep FP120 Cell Disrupter, speed 4.5, QBiogen, MP Biomedicals, France) with glass beads $425-600 \mu \mathrm{m}$ (Sigma-Aldrich) was added to the protocol. $\mathrm{qPCR}$ was performed with primers PRBA388F and PRUN518R on a 7500 Fast real-time PCR instrument (Applied Biosystems, Foster City, CA) using Escherichia coli K12 as standard as described previously. ${ }^{48}$

\section{Flow cytometry}

Lymphocytes from MLN and spleens were prepared as previously described, ${ }^{49}$ stained for regulatory $\mathrm{T}$ cells, and analyzed on an Accuri C6 flow cytometer (Accuri Cytometers, Ann Arbor, MI). T-cell subsets were stained in spleen, colon intra epithelial lymphocytes (IEL) and lamina propria lymphocytes (LPL) and measured on a BDLSRFortessa $^{\mathrm{m}}$ analyzer (BD-Biosciences, Franklin Lakes, NJ). It was not possible to measure iNKT cells in colon IEL and LPL of inoculated mice due to sample limitations. See Supplementary material for detailed description of used antibodies and protocol. See previously published protocol ${ }^{20}$ for gating strategy.

\section{Histological analysis}

Colon was embedded in paraffin and cut in 1-2 $\mu \mathrm{m}$ sections using a histiotome MICROM HM355S (Sakura Finetek Denmark) and stained with hematoxylin and eosin. Each slide was blinded and evaluated by two independent scientists according to mononuclear cell and neutrophil infiltration given the score $0=$ no infiltration, $1=$ mild infiltration, $2=$ moderate infiltration, and $3=$ severe infiltration. Cell turnover was evaluated by the average number of mitotic figures in 10 High Power Fields $(40 \times)$ per slide.

\section{Cytokine analysis}

Enzyme-linked immunosorbant assay (ELISA) was performed to assess the concentrations of IFN $\gamma$, IL1 $\beta$, IL2, IL4, IL5, IL10, IL12p70, IL13, KC/GRO, TNFa, and myeloperoxidase (MPO) in colon and serum from conventional mice together with colonic concentration of IFNY and TNFa in inoculated mice. Furthermore MPO was measured in serum from inoculated mice, but due to sample limitations, it was not possible to measure MPO in colon. See Supplementary material for detailed protocol.

\section{Gene expression analysis}

Colon homogenate was prepared and RNA isolated with MagMAX-96 RNA Isolation Kit (Applied Biosystems), and cDNA synthesis using High-Capacity cDNA Reverse Transcriptase Kit (Applied Biosystems) were conducted as previously noted..$^{50} \mathrm{qPCR}$ was performed using Tagman gene expression assays (Applied biosystems) on a StepOnePlus instrument (Applied Biosystems) using universal fast thermal cycling parameters and TaqMan Fast universal advanced PCR Mastermix (Applied Biosystems). 
See Supplementary material for detailed protocol, running conditions, data analysis, and gene assays.

\section{Statistics}

Statistical analysis was conducted in GraphPad Prism version 7.02 (GraphPad Software, San Diego, CA). Because the vehicle groups and oxazolone groups were not run simultaneously, they were split up in the analysis to avoid time bias, intra-assay and lot variation. Significance was tested by student's $t$-test for parametric data and Mann Whitney's U-test for nonparametric data. Weight change and feces score was tested by two-way ANOVA. Frequencies of euthanasia due to the humane endpoint were compared by Mantel-Cox curve comparison test. Gene expression is given as fold change (RQ) in the plot, but analysis was made on $\Delta C \mathrm{Ct}$ values. $p$ values $<0.05$ were considered significant.

\section{ACKNOWLEDGEMENTS}

Thanks to the NIH Tetramer Core Facility for providing the CD1d tetramer used in this study.

\section{AUTHOR CONTRIBUTIONS}

Study conception and design: LFZ, AKH, DSN, TLH, CHFH; Acquisition of data: LFZ, LK, $\mathrm{PT}, \mathrm{CHFH}$. Analysis and interpretation of data; LFZ, LK, CHFH; Drafting of manuscript: LFZ, LK, PT, CHFH. Critical revision: LFZ, AKH, DSN, TLH, PT, CHFH. Final approval: LFZ, AKH, LK, DSN, TLH, PT, CHFH.

\section{ADDITIONAL INFORMATION}

The online version of this article (https://doi.org/10.1038/s41385-019-0207-8) contains supplementary material, which is available to authorized users.

Competing interests: The authors declare no competing interests.

Publisher's note Springer Nature remains neutral with regard to jurisdictional claims in published maps and institutional affiliations.

\section{REFERENCES}

1. Betran, A. P. et al. The increasing trend in caesarean section rates: global, regional and national estimates: 1990-2014. PloS ONE 11, e0148343 (2016).

2. Lumbiganon, P. et al. Method of delivery and pregnancy outcomes in Asia: the WHO global survey on maternal and perinatal health 2007-08. Lancet 375, 490-499 (2010).

3. Rebelo, F., da Rocha, C. M., Cortes, T. R., Dutra, C. L. \& Kac, G. High cesarean prevalence in a national population-based study in Brazil: the role of private practice. Acta Obstet. Gynecol. Scand. 89, 903-908 (2010).

4. Bager, P., Simonsen, J., Nielsen, N. M. \& Frisch, M. Cesarean section and offspring's risk of inflammatory bowel disease: a national cohort study. Inflamm. Bowel Dis. 18, 857-862 (2012).

5. Sevelsted, A., Stokholm, J., Bonnelykke, K. \& Bisgaard, H. Cesarean section and chronic immune disorders. Pediatrics 135, e92-e98 (2015).

6. Malmborg, P., Bahmanyar, S., Grahnquist, L., Hildebrand, H. \& Montgomery, S. Cesarean section and the risk of pediatric Crohn's disease. Inflamm. Bowel Dis. 18, 703-708 (2012).

7. Sartor, R. B. Mechanisms of disease: pathogenesis of Crohn's disease and ulcerative colitis. Nat. Clin. Pract. Gastroenterol. Hepatol. 3, 390-407 (2006).

8. Sartor, R. B. \& Wu, G. D. Roles for intestinal bacteria, viruses, and fungi in pathogenesis of inflammatory bowel diseases and therapeutic approaches. Gastroenterology 152, 327-339.e324 (2017).

9. Knights, D., Lassen, K. G. \& Xavier, R. J. Advances in inflammatory bowel disease pathogenesis: linking host genetics and the microbiome. Gut 62, 1505-1510 (2013)

10. Elinav, E. et al. NLRP6 inflammasome regulates colonic microbial ecology and risk for colitis. Cell 145, 745-757 (2011).

11. Sellon, R. K. et al. Resident enteric bacteria are necessary for development of spontaneous colitis and immune system activation in interleukin-10-deficient mice. Infect. Immunol. 66, 5224-5231 (1998).

12. Kim, N., Kunisawa, J. Kweon, M. N., Eog Ji, G. \& Kiyono, H. Oral feeding of Bifidobacterium bifidum (BGN4) prevents $\mathrm{CD} 4(+)$ CD45RB(high) $\mathrm{T}$ cell-mediated inflammatory bowel disease by inhibition of disordered T cell activation. Clin. Immunol. 123, 30-39 (2007).

13. Bendtsen, K. M., Fisker, L., Hansen, A. K., Hansen, C. H. \& Nielsen, D. S. The influence of the young microbiome on inflammatory diseases-lessons from animal studies. Birth Defects Res. C. Embryo Today 105, 278-295 (2015).

14. Olszak, T. et al. RS. Microbial exposure during early life has persistent effects on natural killer T cell function. Science 336, 489-493 (2012).

15. Atarashi, K. et al. Treg induction by a rationally selected mixture of Clostridia strains from the human microbiota. Nature 500, 232-236 (2013).

16. Geuking, M. B. et al. Intestinal bacterial colonization induces mutualistic regulatory T cell responses. Immunity 34, 794-806 (2011).

17. Yamada, A. et al. Role of regulatory $T$ cell in the pathogenesis of inflammatory bowel disease. World J. Gastroenterol. 22, 2195-2205 (2016).

18. Powrie, F., Correa-Oliveira, R., Mauze, S. \& Coffman, R. L. Regulatory interactions between CD45RB high and CD45RBlow CD4+T cells are important for the balance between protective and pathogenic cell-mediated immunity. J. Exp. Med. 179, 589-600 (1994).

19. Ponsonby, A. L. et al. Association between early-life factors and risk of child-onset Crohn's disease among Victorian children born 1983-1998: a birth cohort study. Inflamm. Bowel Dis. 15, 858-866 (2009).

20. Zachariassen, L. F. et al. Cesarean section induces microbiota-regulated immune disturbances in C57BL/6 mice. J. Immunol., https://doi.org/10.4049/ jimmunol.1800666 (2018).

21. Hviid, A., Svanstrom, H. \& Frisch, M. Antibiotic use and inflammatory bowel diseases in childhood. Gut 60, 49-54 (2011).

22. Xu, L. et al. Systematic review with meta-analysis: breastfeeding and the risk of Crohn's disease and ulcerative colitis. Aliment. Pharmacol. Ther. 46, 780-789 (2017).

23. Sonntag, B. et al. Preterm birth but not mode of delivery is associated with an increased risk of developing inflammatory bowel disease later in life. Inflamm. Bowel Dis. 13, 1385-1390 (2007).

24. Michielan, A. \& D'Inca, R. Intestinal permeability in inflammatory bowel disease: pathogenesis, clinical evaluation, and therapy of leaky gut. Mediators Inflamm. 2015, 628157 (2015).

25. Zeissig, S. et al. Changes in expression and distribution of claudin 2, 5 and 8 lead to discontinuous tight junctions and barrier dysfunction in active Crohn's disease. Gut 56, 61-72 (2007).

26. Suenaert, P. et al. Anti-tumor necrosis factor treatment restores the gut barrier in Crohn's disease. Am. J. Gastroenterol. 97, 2000-2004 (2002).

27. Vogel, C. \& Marcotte, E. M. Insights into the regulation of protein abundance from proteomic and transcriptomic analyses. Nat. Rev. Genet. 13, 227-232 (2012).

28. Sawant, K. V. et al. Chemokine CXCL1 mediated neutrophil recruitment: Role of glycosaminoglycan interactions. Sci. Rep. 6, 33123 (2016).

29. Aratani, Y. Myeloperoxidase: Its role for host defense, inflammation, and neutrophil function. Arch. Biochem. Biophys. 640, 47-52 (2018).

30. Rakoff-Nahoum, S., Paglino, J., Eslami-Varzaneh, F., Edberg, S. \& Medzhitov, R. Recognition of commensal microflora by toll-like receptors is required for intestinal homeostasis. Cell 118, 229-241 (2004).

31. Takedatsu, H. et al. TL1A (TNFSF15) regulates the development of chronic colitis by modulating both T-helper 1 and T-helper 17 activation. Gastroenterology 135, 552-567 (2008).

32. Jia, L. G. et al. A Novel Role for TL1A/DR3 in Protection against Intestinal Injury and Infection. J. Immunol. 197, (377-386 (2016).

33. Izcue, A., Coombes, J. L. \& Powrie, F. Regulatory T cells suppress systemic and mucosal immune activation to control intestinal inflammation. Immunol. Rev. 212, 256-271 (2006)

34. $\mathrm{Yu}, \mathrm{L}$. et al. CD69 enhances immunosuppressive function of regulatory T-cells and attenuates colitis by prompting IL-10 production. Cell Death Dis. 9, 905 (2018).

35. Braun, A. et al. Integrin alphaE(CD103) is involved in regulatory T-cell function in allergic contact hypersensitivity. J. Investig. Dermatol. 135, 2982-2991 (2015).

36. Hansen, C. H. et al. Mode of delivery shapes gut colonization pattern and modulates regulatory immunity in mice. J. Immunol. 193, 1213-1222 (2014).

37. Martinez, K. A. 2nd et al. Increased weight gain by C-section: Functional significance of the primordial microbiome. Sci. Adv. 3, eaao1874 (2017).

38. Larsen, J. M. The immune response to Prevotella bacteria in chronic inflammatory disease. Immunology 151, 363-374 (2017).

39. Dziarski, R., Park, S. Y., Kashyap, D. R., Dowd, S. E. \& Gupta, D. Pglyrp-regulated gut microflora Prevotella falsenii, Parabacteroides distasonis and Bacteroides eggerthii enhance and Alistipes finegoldii attenuates colitis in mice. PloS ONE 11, e0146162 (2016).

40. Boksa, P., Zhang, Y. \& Bestawros, A. Dopamine D1 receptor changes due to caesarean section birth: effects of anesthesia, developmental time course, and functional consequences. Exp. Neurol. 175, 388-397 (2002). 
41. Zanardo, V. et al. Neonatal respiratory morbidity risk and mode of delivery at term: influence of timing of elective caesarean delivery. Acta Paediatr. 93, 643-647 (2004).

42. Bessler, H. et al. Labor affects cytokine production in newborns. Am. J. Reprod. Immunol. 39, 27-32 (1998).

43. Ly, N. P. et al. Mode of delivery and cord blood cytokines: a birth cohort study. Clin. Mol. Allergy. 4, 13 (2006).

44. Thilaganathan, B., Meher-Homji, N. \& Nicolaides, K. H. Labor: an immunologically beneficial process for the neonate. Am. J. Obstet. Gynecol. 171, 1271-1272 (1994).

45. Marafini, I., Sedda, S., Dinallo, V. \& Monteleone, G. Inflammatory cytokines: from discoveries to therapies in IBD. Exp Opin. Biol. Ther., 1-11, https://doi.org/10.1080/ 14712598.2019.1652267 (2019).

46. MacDonald, T. T., Hutchings, P., Choy, M. Y., Murch, S. \& Cooke, A. Tumour necrosis factor-alpha and interferon-gamma production measured at the single cell level in normal and inflamed human intestine. Clin. Exp. Immunol. 81, 301-305 (1990).

47. Holgersen, K., Kvist, P. H., Markholst, H., Hansen, A. K. \& Holm, T. L. Characterisation of enterocolitis in the piroxicam-accelerated interleukin-10 knock out mouse-a model mimicking inflammatory bowel disease. J. Crohn's. Colitis 8, 147-160 (2014).

48. Nguyen, D. N. et al. Oral antibiotics increase blood neutrophil maturation and reduce bacteremia and necrotizing enterocolitis in the immediate postnatal period of preterm pigs. Innate Immun. 22, 51-62 (2016).

49. Hansen, C. H. et al. Early life treatment with vancomycin propagates Akkermansia muciniphila and reduces diabetes incidence in the NOD mouse. Diabetologia 55, 2285-2294 (2012).

50. Hansen, C. H. et al. Dietary xylooligosaccharide downregulates IFN-gamma and the low-grade inflammatory cytokine IL-1beta systemically in mice. J. Nutr. 143, 533-540 (2013). 